\title{
The morphology, mechanical properties and ageing behavior of porous injection molded starch-based blends for tissue engineering scaffolding
}

\author{
Nuno M. Neves*, A. Kouyumdzhiev, Rui L. Reis \\ 3B's Research Group-Biomaterials, Biodegradables and Biomimetics, University of Minho, Campus de Gualtar, 4710-057 Braga, Portugal \\ Department of Polymer Engineering, University of Minho, Campus de Azurém, 4800-058 Guimarães, Portugal
}

Available online 9 March 2005

\begin{abstract}
One important parameter in the tissue engineering of hard tissues is the scaffold. A scaffold is a support in which cells are seeded and that should create the adequate environment for the cells to attach and proliferate. Furthermore the scaffold should allow the flow of an appropriate culture media, providing nutrients to the cells and simultaneously removing the metabolites resulting from the cells activity. One of the possibilities is to obtain solid foamed structures that will enable the cells to attach, spread into the inner surfaces and start to produce extracellular matrix. Ideally, if the scaffold is produced from a biodegradable material, it should degrade at a pace that is in phase with the formation of the new tissue.

In this work it was studied the production of porous structures from biodegradable polymers for use as scaffolds for bone tissue engineering. Two materials were studied, starch compounded with poly(ethylene-vinyl-alcohol) (SEVA-C) and starch with poly(lactic acid) (SPLA). The porous structures were obtained by injection molding with a blowing agent to control the porosity, interconnectivity and degradation rate. In previous attempts, the current starch compounds proved to be very difficult to process by this method. This study includes the characterization of the mechanical properties, water absorption and of the degradation kinetics of the 3-D porous structures.

Two starch-based biodegradable 3D porous structures were successfully processed in conventional injection molding and the foaming was obtained by means of the use of a blowing agent. The mechanical properties are very promising as well as the improved degradation kinetics when compared with the synthetic polymers alone, although the degree of porosity and of interconnectivity needs to be improved in further work.
\end{abstract}

(C) 2005 Elsevier B.V. All rights reserved.

Keywords: Starch; Injection molding with blowing agent; Scaffolds for tissue engineering; Biodegradable polymers; Cellular material

\section{Introduction}

Both injection molding and extrusion of materials containing blowing agents (BA) are important industrial processing technologies for obtaining cellular foamed products $[1,2]$. Studies of bubble development and morphology of polymers in foaming extrusion processes and the effect of processing variables on the quality of the produced foams have been reported by several authors [3-5].

An important aspect of foam extrusion is the mechanics of bubble growth in the polymeric matrix. A number

\footnotetext{
* Corresponding author.

E-mail address: nuno@dep.uminho.pt (N.M. Neves).
}

of experimental studies of bubble growth during the microcellular foaming process and the influence of temperature, injection pressure, molecular weight and the nature of the blowing agent have been reported previously (e.g. [6-8]).

The advent of tissue engineering has been motivated by the shortage of transplantation organs and by the challenge of producing tissue substitutes, which could be implanted into the body to repair it or to restore its function. In fact, the goal is to promote the regeneration of the structural features and physiological functionalities of the living tissues $[9,10]$. This multidisciplinary research area combines the science and technology of materials, materials engineering, surface science, chemistry and biology to obtain hybrid materials to be used in regenerative medicine. 
There are examples of virtually all kinds of biomaterials used in orthopedic applications including metals, alloys, ceramics, modified natural biomaterials, synthetic polymers and composites consisting of different combinations of these materials [11-13]. The first requirement for any synthetic material used for implantation in the body is that it should be biocompatible [14-16] and not causing any significant inflammatory reaction in the body.

There are many potential applications for synthetic or natural polymers both as implants and as medical devices in clinical medicine. The primary application areas include cardiovascular [17] as soft tissue replacements or dental [18] and orthopedic [19] in the case of hard tissues.

Biodegradable surgical implants are ideal solutions for temporary internal fixation when tissue has been traumatized since the surgical procedure for implant removal is avoided [12,20,21]. At an early stage of healing, the biodegradable implant preserves the structure of the tissue and its function. In time, the implant gradually decomposes and the functions are progressively transferred to the healed tissue. The time necessary for this process depends mainly on the type of polymer and its molecular weight and on the specific biochemical and mechanical environment inside the body.

There are two main stages of resorption of a biodegradable material when subjected to physiological medium: water infiltrates the polymer and attacks the polymer chains leading to chain scission by hydrolysis; the polymer loses mass and the low molecular chains are extracted and phagocytized by macrophages (e.g. [22]). The chain scission process may be also affected by enzymatic activity and by the action of aggressive fluids of the organism. It is paramount to ensure that both the materials and its residues are harmless both for the tissue being regenerated and to the surrounding tissues. Previous studies using starch based compounds showed that the starch phase in the compound, being more hydrophilic, enhances the water infiltration process $[23,24]$. The presence of starch positively affects the kinetics of degradation of synthetic biodegradable polymers.

It is well known that isolated cells are not able to reconstruct the tissue from where the cells are extracted. A great scientific effort is being directed into the design and the production of constructs that assist the cells in tissue regeneration. Thus, a very important stage of tissue engineering for hard tissues is the design and processing of scaffolds with adequate 3D geometries. The requirements of those constructs include a controlled porosity (including pore size and inner interconnectivity). Furthermore, the construct should have adequate mechanical properties both because the living cells require mechanical stimuli to promote tissue regeneration $[25,26]$ and for obtaining functional characteristics after implantation. Additionally, the surface chemistry and topography may play an important role in recruiting proteins and interacting with living cells. It has been shown that starch based materials have adequate biocompatibility, showing no cytotoxic harmful behavior when in contact with cells (e.g. [27]), thus being very promising for tissue engineering applications. Furthermore, its compounding with polylactic acid would allow obtaining improved mechanical properties in the scaffold which is very relevant for load bearing applications.

In this work two kinds of biodegradable polymers were used in order to produce porous scaffolds. The processing technique was conventional injection molding process using a blowing agent. The goal is to obtain novel 3D porous constructs with potential to be used in bone tissue engineering combining the biocompatibility and degradation properties of starch with the superior mechanical properties of a polylactic acid and poly(ethylene vinyl alcohol). The kinetics of the degradation was studied by immersion in isotonic saline solution for periods of up to 30 days to characterize the ageing stability of the 3D constructs.

\section{Materials and methods}

The polymers used in this work were based on blends of corn starch with: (i) ethylene vinyl alcohol (SEVA-C 50/50 wt.\%); (ii) 70 wt.\% poly(lactic acid) (SPLA 70). All the materials were supplied by Novamont, Italy.

The selected blowing agent was based on azo-dicarbonamide with the following structural formula:

$\mathrm{H}_{2} \mathrm{~N}-\mathrm{OC}-\mathrm{N}=\mathrm{N}-\mathrm{CO}-\mathrm{N}_{2} \mathrm{H}$

and molecular weight 116 (trade name Azobul K3, Elf Atochem S.A., France).

The work included the development and preparation of biodegradable polymeric scaffolds with $0.10 \%, 0.20 \%$ and $0.30 \%$ of BA and biopolymers. The polymers and the BA were previously premixed together in a rotating drum. Materials were then molded in an injection molding machine, Krauss Maffei KM 60-120A, to produce discs with $60 \mathrm{~mm}$ diameter and $16 \mathrm{~mm}$ wall thickness. The large wall thickness was intended to facilitate the expansion of the blowing agent and leading to a more controlled porosity distribution.

Samples obtained by the injection molding process were characterized in terms on their morphology, mechanical properties and degradation behavior. The cytotoxicity of these polymers was previously tested and the results showed a non-cytotoxic behavior $[27,28]$.

Scanning electron microscopy (JSM-6100, Mountain View, Ca, USA) was used as an indication of the inner porosity and interconnectivity of the scaffolds.

The developed materials were mechanically tested on compression experiments in an Instron 4505 universal tensile testing machine using a load cell of $50 \mathrm{kN}$. The tests were carried out in a controlled environment $\left(23{ }^{\circ} \mathrm{C}\right.$ and $55 \% \mathrm{RH})$ and at a cross-head speed of $2 \mathrm{~mm} / \mathrm{min}$. A minimum of eight specimens was tested for each condition. 


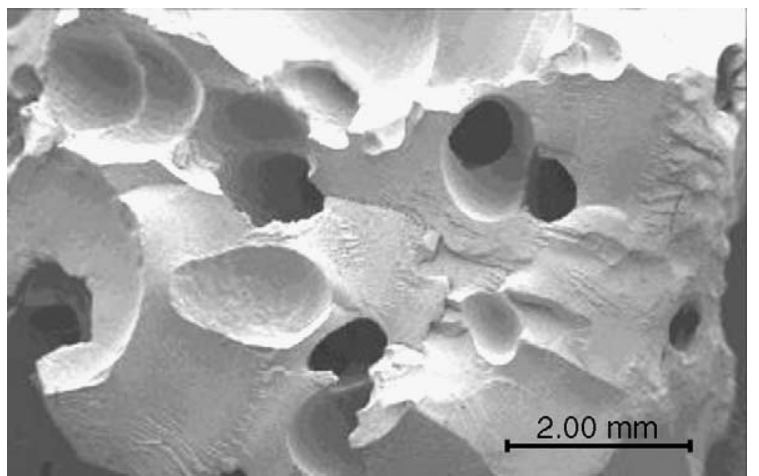

Fig. 1. Typical microstructure obtained in scaffolds of SPLA70 with $0.2 \%$ of blowing agent.

The degradation behavior of the polymers used in this study was characterized after several pre-fixed ageing periods $(1,3,7,14$ and 30 days) in isotonic saline solution $(0.154 \mathrm{M}$ of $\mathrm{NaCl})$. Before being put into the solution, the specimens were dried in an oven at $55{ }^{\circ} \mathrm{C}$ until the equilibrium was reached (assessed by constant weight in two consecutive readings). After being removed from the solution, the samples were dried again at the same temperature, until they reached the equilibrium with the humidity of the environment. Later on, water uptake and weight loss calculations were done. A different set of specimens was dried up at the same conditions, to be tested in compression to evaluate the evolution of the mechanical properties as a function of the degradation time.

\section{Results and discussion}

\subsection{Morphology of the porous structure}

The porous structures obtained in injection molding of the two blends previously mixed with blowing agent, results from the thermal decomposition of the $\mathrm{BA}$ during processing and releasing of $\mathrm{CO}_{2}$ and $\mathrm{H}_{2} \mathrm{O}$. The pores are formed in the bulk of the polymer and depending on the

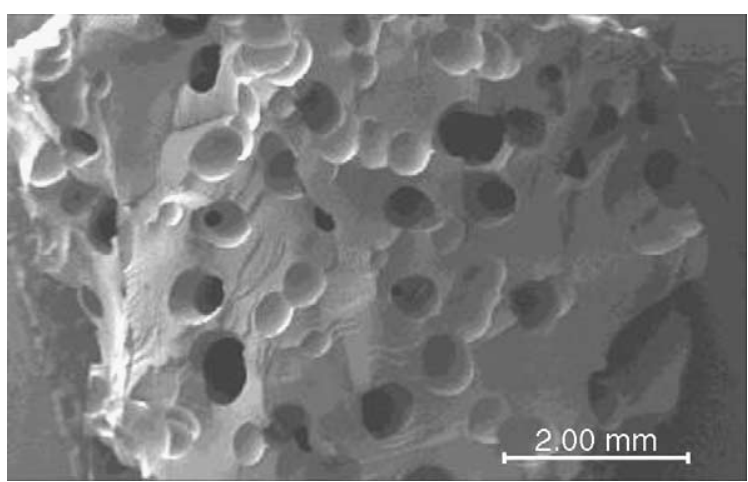

Fig. 2. Typical microstructure obtained in scaffolds of SPLA 70 with $0.3 \%$ of blowing agent.

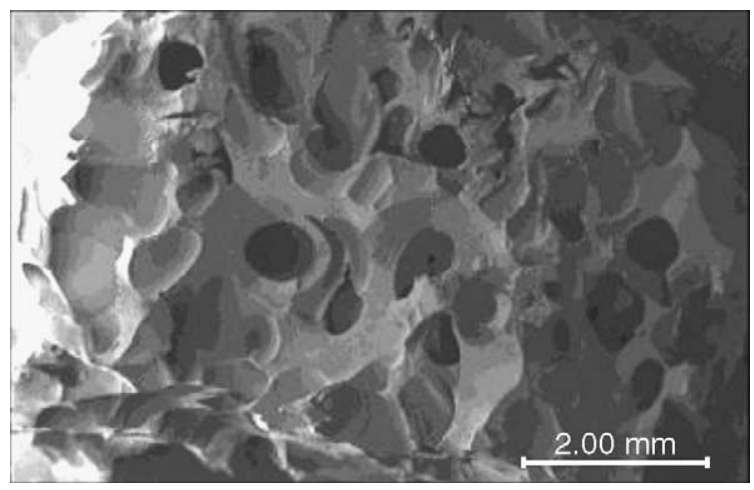

Fig. 3. Typical microstructure obtained in scaffolds of SEVA-C with $0.2 \%$ of blowing agent.

amount of blowing agent and the specific molding conditions used it is possible to tune its structure and dimensions. The molded part is a structure in which the bulk of the material has porosity, whereas the skin is solid (unfoamed) material $[29,30]$. This is a result of the faster cooling of the material in contact with the cold mold walls.

The scaffolds obtained from SPLA70 by injection molding process with BA (Fig. 1) have pore size roughly between 380 and $500 \mu \mathrm{m}$, but the interconnectivity is rather poor. With the same compound but with different percentage of BA and different molding conditions (Fig. 2) it can be seen that it was possible to obtain higher levels of porosity and some interconnectivity on the porous structures.

It is possible to observe that the porosity of the SEVA-C scaffold is larger than the porosity obtained with SPLA 70, both injected with the same amounts of blowing agent (Figs. 1 and 3). Using the lowest amount of blowing agent in SEVA-C (Fig. 4) and adequate processing conditions it is possible to optimize the porosity, improve the interconnectivity and obtain smaller pore sizes. The pore sizes obtained with those conditions range between 100 and $400 \mu \mathrm{m}$. Those pore dimensions are within the range of sizes that lead to the best results for bone cell culture and bone tissue ingrowth, as reported previously [31-33].

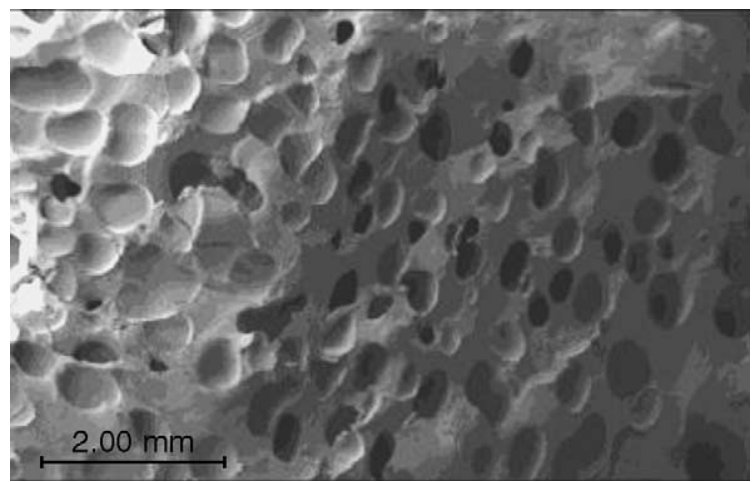

Fig. 4. Typical microstructure obtained in scaffolds of SEVA-C with $0.1 \%$ of blowing agent. 
The density of the specimens was measured in order to confirm the qualitative porosity assessment of the scaffolds by SEM. For SEVA-C it is approximately $0.7 \mathrm{~g} / \mathrm{cm}^{3}$ and for SPLA 70 approximately $0.8 \mathrm{~g} / \mathrm{cm}^{3}$. The values of porosity are mainly controlled by the amount of blowing agent used and by the fine tuning of the processing conditions.

\subsection{Mechanical properties}

The two blends proposed for producing scaffolds by injection molding with blowing agents, show different compression properties, with its largest modulus ranging from 200 to $800 \mathrm{MPa}$.

In Table 1 it is possible to observe that the SPLA 70 samples consistently show higher mechanical properties than SEVA-C samples. The same trend is observed in the aged specimens after immersion in isotonic saline solution. SPLA 70 samples have about four times higher the value of the compression modulus when compared with SEVA-C samples. This could be explained by the stiffness of the synthetic polymer and by the concentration of starch being larger in SEVA-C than in SPLA 70. Furthermore, SPLA 70 samples showed more controlled porosity for most injection molding parts, even if the average amount of porosity obtained for both materials does not differ significantly (Figs. 5 and 6). However, it can also be observed in the same pictures that the size of the porosity can be very different.

The compression strength results for both materials show the same trends as observed in the compression modulus. SPLA70 specimens have values of strength about twice larger than SEVA-C. The strain at break of SPLA 70 scaffolds is considerably lower than the ones of SEVA-C. The same trend could be observed with immersed samples. Again here the difference may be attributed both to the different properties of the synthetic

Table 1

Mechanical properties including compression modulus, strength and strain at break of SEVA-C and SPLA70 scaffolds and the ageing effect of immersion in isotonic saline solution

\begin{tabular}{lllllc}
\hline Material & $\begin{array}{l}\text { Immersion } \\
\text { time (days) }\end{array}$ & $\begin{array}{l}\text { Blowing } \\
\text { agent } \\
(\%)\end{array}$ & $\begin{array}{l}\text { Compression } \\
\text { modulus, } \\
\text { MPa }\end{array}$ & $\begin{array}{l}\text { Compression } \\
\text { strength, } \\
\text { MPa }\end{array}$ & $\begin{array}{l}\text { Strain at } \\
\text { break, } \\
\%\end{array}$ \\
\hline SEVA-C & 0 & 0.1 & $200 \pm 45$ & $14 \pm 5$ & $30 \pm 9$ \\
& & 0.2 & $192 \pm 29$ & $14 \pm 2$ & $30 \pm 8$ \\
& 3 & 0.1 & $22 \pm 3$ & $5 \pm 3$ & $35 \pm 8$ \\
& \multirow{2}{*}{14} & 0.2 & $43 \pm 11$ & $6 \pm 3$ & $31 \pm 2$ \\
& & 0.1 & $39 \pm 9$ & $4 \pm 2$ & $32 \pm 2$ \\
SPLA 70 & 0 & 0.2 & $40 \pm 13$ & $5 \pm 2$ & $32 \pm 4$ \\
& & 0.2 & $785 \pm 88$ & $29 \pm 10$ & $17 \pm 2$ \\
& \multirow{2}{*}{3} & 0.3 & $896 \pm 95$ & $23 \pm 4$ & $10 \pm 5$ \\
& \multirow{2}{*}{14} & 0.2 & $286 \pm 83$ & $11 \pm 3$ & $17 \pm 7$ \\
& 0.3 & $210 \pm 65$ & $8 \pm 3$ & $13 \pm 3$ \\
& 0.2 & $267 \pm 36$ & $10 \pm 3$ & $15 \pm 6$ \\
& 0.3 & $240 \pm 51$ & $8 \pm 3$ & $9 \pm 4$ \\
\hline
\end{tabular}

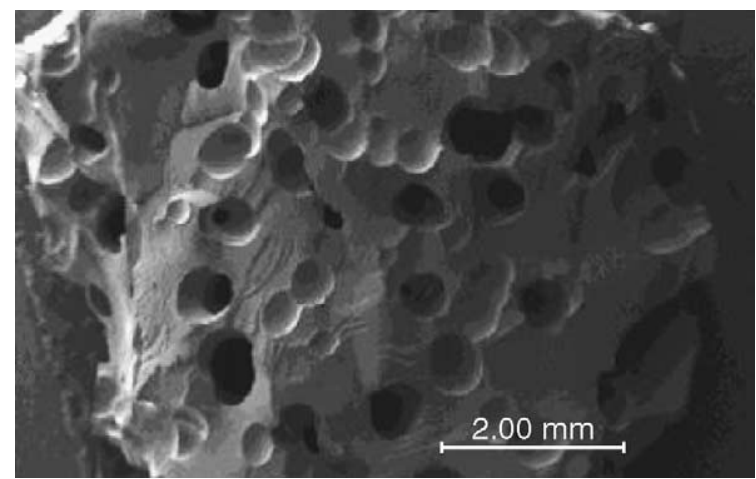

Fig. 5. Porosity of SPLA 70 scaffolds.

phase and to the microstructure of the scaffolds. However, it seems that the mechanical properties of SEVA-C are much more sensitive to the immersion in isotonic saline solution.

The scaffolds obtained from SEVA-C and SPLA70 blends show very promising mechanical properties compared even with the same blends, but processed with other techniques such as solvent casting or particle leaching [34].

The compression modulus of the scaffolds seems to be affected by the amount of the blowing agent used and on the processing conditions, particularly in the case of SPLA 70. The mechanical properties might be further improved by changing the type and percentage of the blowing agent and further improve the porosity and interconnectivity of the scaffolds.

Specimens from both polymers were immersed in isotonic saline solution (ISS), for periods of 3 and 14 days. After equilibrating their humidity, they were mechanically tested. The results for both materials show lower values of mechanical properties after the periods of immersion (Table 1). This is expected since the presence of water has a plasticising effect over the material leading to increased compliance (lower modulus). In fact, upon immersion after 3 days, the values of stiffness have a 10 fold decrease in the case of SEVA-C being less dramatic the decrease in stiffness in the case of SPLA 70. The degradation leads in either blends to loss of low molecular weight material enhancing

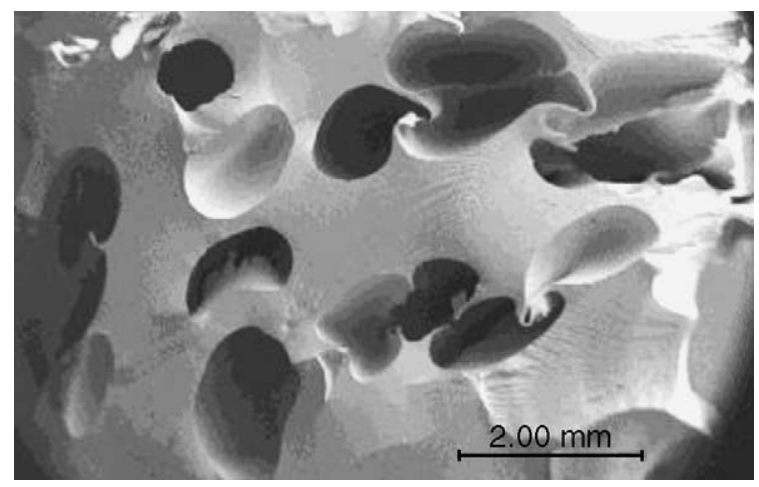

Fig. 6. Porosity of SEVA-C scaffolds. 


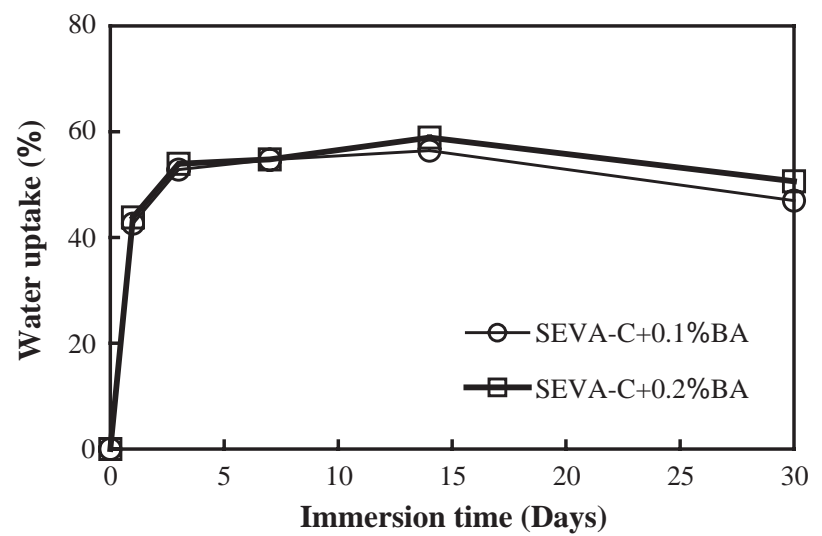

Fig. 7. Water uptake of SEVA-C porous scaffolds for different periods of immersion in isotonic saline solution.

the microporosity of the materials and reducing its strength. Longer periods of immersion do not show such intense variation of mechanical properties.

\subsection{Degradation behavior}

Observing Figs. 7 and 8, it is possible to say that for both polymeric blends the water uptake value increases with increasing the amount of BA used. The differences are related with the fact that the percentage of water uptake for SEVA-C decreases for longer immersion periods while the opposite is observed with SPLA70. The water uptake of SEVA-C reaches values of around $60 \%$, while for SPLA70 has a maximum at around $45 \%$. Weight loss experiments confirm this trend (Figs. 9 and 10). It was observed for both blends that higher amounts of blowing agent lead to higher values of weight loss, possibly due to the larger inner porosity and interconnectivity between the pores. SPLA70 based scaffolds underwent higher weight loss than the SEVA-C blend, possibly due to the nature of the different synthetic component of the two starch-based compounds and their distinct morphology. It was also observed that the compact outer layer of the specimens tends to start degrading faster and microporosity appears after shorter immersion periods. This process of degradation is beneficial

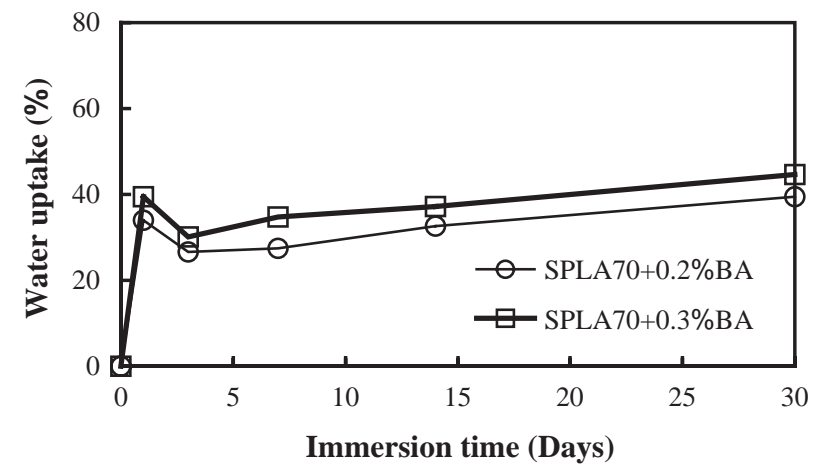

Fig. 8. Water uptake of SPLA 70 porous scaffolds for different periods of immersion in isotonic saline solution.

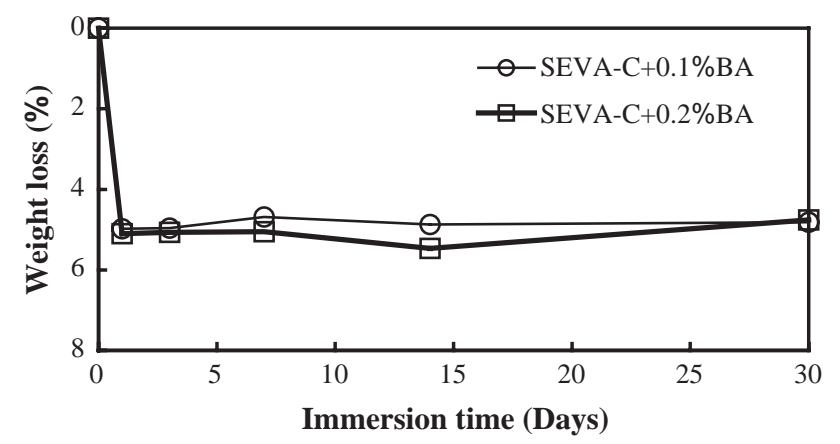

Fig. 9. Weight loss of SEVA-C porous scaffolds for different periods of immersion in isotonic saline solution.

since it provides a pathway for the cells and nutrients to penetrate into the inner core of the material.

The injection molding with blowing agent does not influence the non-cytotoxicity of the starch-based polymers, as reported in previous works [35].

\section{Conclusions}

It was possible to obtain porous structures from the two starch-based blends. Injection molding with a blowing agent is an adequate processing method for the production and control of the morphology of porous biodegradable polymers.

The pore size and interconnectivity of the pores is sensitive to the processing parameters under study. The type and geometry of the pores is similar of both materials, but SPLA 70 has more controlled dimensions and distribution of the pores having also higher interconnectivity than SEVA-C.

The mechanical properties of SPLA 70 scaffolds are consistently larger than of the SEVA-C ones. SPLA 70 has more than four times higher compression modulus and less strain at break.

Weight loss of the scaffolds depends mainly on the processing condition and it is similar for both materials but the weight loss is lower in the case of SEVA-C samples.

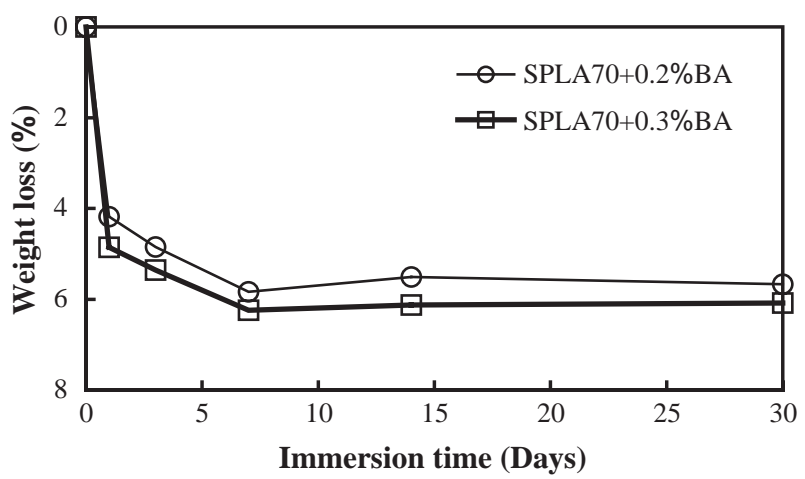

Fig. 10. Weight loss of SPLA 70 porous scaffolds for different periods of immersion in isotonic saline solution. 
Porous structures obtained from SPLA 70 and SEVA-C using injection molding with blowing agents, even if requiring further optimization particularly increasing its porosity and interconnectivity, may constitute an important alternative to other technologies in producing scaffolds for tissue engineering.

\section{References}

[1] D.M. Bigg, J.R. Preston, D. Banner, Polym. Eng. Sci. 16 (1976) 706.

[2] H. Gonzales, J. Cell. Plast. 12 (1976) 49.

[3] Y. Oyangi, J.L. White, J. Appl. Polym. Sci. 23 (1979) 1013.

[4] C.D. Han, Y.W. Kim, K.D. Malhatra, J. Appl. Polym. Sci. 20 (1976) 1583.

[5] C.D. Han, C.A. Villamizar, Polym. Eng. Sci. 18 (1978) 687.

[6] N.S. Ramesh, D.H. Rasmussen, G.A. Campbell, Polym. Eng. Sci. 31 (1991) 1657.

[7] Y.Q. Meng, D.M. Wu, Appl. Phys. Lett., A 327 (2004) 61.

[8] M. Hatanaka, H. Saito, Macromolecules 37 (2004) 7358.

[9] L.E. Freed, G. Vunjak-Novakovic, Adv. Drug Deliv. Rev. 33 (1998) $15-30$.

[10] R.C. Thomson, M.C. Wake, M. Yaszemski, A.G. Mikos, Adv. Polym. Sci. 122 (1995) 247.

[11] D.F. Williams, Prog. Biomed. Eng. 4 (1987).

[12] D.F. Williams, in: G. Allen, J.S. Bevington, G.C. Eastmond, A. Ledwith, S. Russo, P. Sigwolt (Eds.), Comprehensive Polymer Science, vol. 6, Pergamon Press, Oxford, 1989, p. 607.

[13] B.D. Ratner, in: G. Allen, J.C. Bevington, S.L. Aggarwal (Eds.), Comprehensive Polymer Science, Pergamon Press, Oxford, 1989, p. 201.

[14] W. Bonfield, in: J.E. Lemons (Ed.), Ann. NY Acad. Sci., 523, 1988, pp. 173.
[15] M.E. Gomes, R.L. Reis, A.M. Cunha, C.A. Blitterswijk, J.D. de Bruijn, Biomaterials 22 (2001) 1911.

[16] A.P. Marques, R.L. Reis, J.A. Hunt, J. Mater. Sci., Mater. Med. 14 (2003) 167.

[17] D.L. Salzmann, L.B. Kleinert, S.S. Berman, S.K. Williams, Card. Pathol. 8 (1999) 63.

[18] M.A. Freilich, J.P. Duncan, E.K. Alarcon, K.A. Eckrote, A.J. Goldberg, J. Prosthet. Dent. 88 (2002) 449.

[19] O. Böstman, H. Pihlajamäki, Biomaterials 21 (2000) 2615.

[20] P.U. Rokkanen, Ann. Med. 23 (1991) 109.

[21] J.M. Anderson, Q.H. Zhao, MRS Bull. (1991) 75-77.

[22] G. Schwach, M. Vert, Int. J. Biol. Macromol. 25 (1999) 283.

[23] R.L. Reis, S.C Mendes, A.M. Cunha, M.J. Bevis, Polym. Int. 43 (1997) 347.

[24] R.L. Reis, A.M. Cunha, J. Appl. Med. Polym. 4 (2000) 1.

[25] R. Langer, J. Control. Release (1999) 7-11.

[26] R. Thomson, M. Yaszemski, A. Mikos, in: R. Lanza, R. Langer, W. Chick (Eds.), Principles of tissue engineering, Academic Press, New York, 1997, p. 263.

[27] A.J. Salgado, M.E. Gomes, A. Chou, O.P. Coutinho, R.L. Reis, D.W. Hutmacher, Mater. Sci. Eng., C 20 (2002) 27.

[28] M.E. Gomes, R.L. Reis, A.M. Cunha, C.A. Blitterwijk, J.D. de Bruijn, Biomaterials 22 (2001) 1017.

[29] G.W. Brewer, Properties of Thermoplastics Structural Foams, Engineered Materials Handbook, vol. 2, ASM International, New York, 1989, p. 508.

[30] D.V. Khakhar, K.V. Joseph, Polym. Eng. Sci. 34 (1994) 726.

[31] L. Lu, A. Mikos, MRS Bull. 21 (1996) 28.

[32] H. Kim, J. Smith, R. Valentini, Tissue Eng. 4 (1998) 35.

[33] K. Whang, K.E. Healy, D.R. Elenz, Tissue Eng. 5 (1999) 35.

[34] M.E. Gomes, J.S. Godinho, D. Tchalamov, A.M. Cunha, R.L. Reis, Mater. Sci. Eng., C 20 (2002) 19.

[35] S.C. Mendes, R.L. Reis, Y.P. Bovell, A.M. Cunha, C.A. van Blitterswijk, J.D. de Bruijn, Biomaterials 22 (2001) 2057. 